\title{
Ciliary Body Tumour Occurring in a Nigerian - A case report
}

\author{
ON Okonkwo MD, FRCSEd, DRCOphth; AO Hassan MD, FRCS, FRCOpth FWACS; O Oderinlo MD, \\ FRCSEd, DRCOphth; F Oluyadi MD, FNMC, FWACS; AO Ogunro MD, FWACS; M Ulaikere MD, \\ FWACS, FNMC; A Harriman MD, MRCOphth; S Oke MD, DWACS, MCEH \\ Eye Foundation Retina Institute, Ijebu Imushin, Ogun State
}

\section{SUMMARY}

Objective: To report the rare case of a 33-year-old female Nigerian who presented to our retina clinic with a chronic total retinal detachment and visual acuity of no light perception in her left eye. Re-attachment surgery in the eye was not attempted as prognosis for return of vision was poor. She was later noted to harbor a progressively enlarging ciliary body mass in the inferotemporal quadrant.

Method: An observational case report was performed, with documentation of findings as patient was seen in the clinic.

Conclusion: This is the first report of a ciliary body mass lesion in a Nigerian. The ciliary body mass could be a ciliary melanoma (ciliary body lesion being rarer than a choroidal mass) but other benign differential diagnoses must be borne in mind. Enucleation and histological assessment of the specimen will give the definitive diagnosis. Difficulties with patient acceptance of enucleating a non seeing eye though harboring a potentially harmful disease still abound in our environment.

Key words: ciliary body melanoma, retinal detachment, sentinel vessels.

\section{BACKGROUND}

Ciliary body melanoma is a subtype of the uveal melanomas. Uveal melanomas are some of the commonest primary intraocular malignancies found in Caucasians. ${ }^{1}$ Uveal melanomas are classified as anterior uveal melanoma when arising from the iris and as posterior uveal melanoma when arising from the ciliary body and choroid.

Ciliary body melanoma is an infrequent tumour. It is encountered less frequently than choroidal melanoma with a ratio of occurrence of $1: 10$ to that in the choroids. ${ }^{2}$ Nonetheless, uveal melanomas are the most common primary malignant tumours of the ciliary body. ${ }^{2}$

Uveal melanomas, including ciliary body melanomas are mostly a disease of Caucasians, particularly those of northern European descent. It is rarely seen among non white people. The incidence of ocular melanoma amongst black peoples is extremely rare. ${ }^{3}$

\section{CASE REPORT}

A 33-year old female Nigerian presented to the clinic with a one-year history of poor vision and seeing of flashes of light in the left eye. The flashes of light predated the visual loss in the left eye. She was referred to our clinic from another eye department after evaluation and investigation. The investigation included a B-scan ultrasonography, after which she was diagnosed as a case of left eye posterior uveitis and retinal detachment.

When she was seen, ocular examination revealed that the visual acuity was $6 / 5$ in the right eye and there was no light perception in the left eye. The anterior segment was normal in both eyes except for the presence of an afferent pupillary defect (APD) in the left eye. Intraocular pressure was right eye $18 \mathrm{mmHg}$ and left eye $16 \mathrm{mmHg}$.

Dilated fundus examination in the right eye revealed a flat retina with cup to disc ratio 0.4 . The optic disc was cupped, with normal appearing vessels and macula.

In the left eye, a dilated fundus examination revealed a total funnel shaped retinal detachment. There was no obvious retinal break seen, though a scleral depression examination was not done to observe the extreme peripheral retina. A rhegmatogenous retinal detachment, though assumed, could not be confirmed.

However, in view of the no light perception vision in the eye, further evaluation was not considered necessary as prognosis for visual return following surgery was extremely poor. A conservative approach to her management was therefore adopted.

She was counselled on the need to care for her right eye, in which she was still able to see.

A right eye central visual field examination was carried out to rule out glaucomatous optic nerve disease. The central visual field exam turned out to be normal. 
She was seen for a routine clinic visit, six months after her initial visit. There were no additional complaints. Her visual acuity remained unchanged in both eyes. She was noted to have prominent and dilated deep episcleral vessels localized to the inferotemporal region of the external left eye. These vessels did not blanch with the use of $2.5 \%$ phenylephrine. There were no such episcleral vessels in the inferonasal part of the left eye and the right eye.

A large, dome shaped, non transilluminating, solid, brownish pigmented mass lesion could be seen in the posterior chamber extending from the region of the ciliary body on a dilated funduscopy (figs 1 and 2). The tumour was localized to the inferotemporal region and seemed to encroach on a yet clear crystalline lens. The lens was displaced slightly upwards by this growing tumour.

A digital B-scan ultrasound examination revealed a solid, echogenic mass originating from the ciliary body region with early posterior extension. A funnel-shaped total retinal detachment was also evident (figs 3 and 4 ).

At that time, the patient was 4 months pregnant. She was to have a systemic work-up. This was meant to rule out the rare possibility of primary extra-ocular malignancy, with presenting intraocular metastasis and the possibility of systemic metastasis of a uveal melanoma.

The patient was advised that enucleation of the left globe and histological study would be the best line of management. The reasons for this included:

I. the fact that the left eye was a non seeing eye.

ii. The characteristics of the tumour were very suggestive of a malignant growth (especially with the dilated episcleral vessels-sentinel vessels and solid nature of the mass), refer to picture and ultrasound scan.

iii. The rate of growth of the tumour and its size within the 6-month duration from initial presentation to the clinic further suggested malignant potential and a risk of extrascleral extension which further decreases the prognosis for the survival of the patient.

iv. Lastly, the finding of retinal detachment in association with a uveal tumour points to a potentially malignant intraocular lesion, rather than a benign lesion.

The patient, however, elected to have a conservative management and defaulted on her clinic appointments.

She was seen again several months after delivery, but after an initial consent to enucleating the left eye, she once again defaulted on her appointment.

\section{DISCUSSION}

There have been previous published reports of posterior uveal melanoma occurring in non-whites. Some of these reports have been in black patients. ${ }^{3-8}$
It has been reported that uveal melanoma is slightly more common in men of all age groups, except for 20-39 years, where a small predilection exists for women. ${ }^{2}$ It is important to note that the patient being reported is 33 years and falls within the 20-39 year age bracket in which women are known to have a predilection for uveal melanoma in Caucasian populations.

There has been a report of ocular melanoma occurring in a 78-year-old female Nigerian by a group in Benin. ${ }^{9}$ In this case the ocular melanoma presented as panophthalmitis, and was classified by the group as a masquerade syndrome. Conjunctival melanoma has also been reported in a 55-yearold male Nigerian. ${ }^{10}$ There has been no published report of a ciliary body tumour occurring in a Nigerian (using the MEDLINE and PubMed search).

This patient presented with a retinal detachment and was then noted to have a ciliary body mass. The typical symptoms most commonly noted by ciliary body melanoma upon presentation are visual loss, photopsia, or visual field alterations. Ciliary body melanomas may be asymptomatic in their early stages, as they can remain hidden behind the iris. ${ }^{11}$ Ciliary body melanomas are not usually visible unless the pupil is widely dilated as shown in our case (see fig 1). Furthermore, as shown in this case, the initial sign of a ciliary body melanoma may be dilated episcleral sentinel vessels in the quadrant harbouring the tumour. ${ }^{11}$ This patient had such sentinel vessels in the inferotemporal bulbar region harbouring the ciliary body mass (see fig 2, with the inferior bulbar conjunctiva exposed).

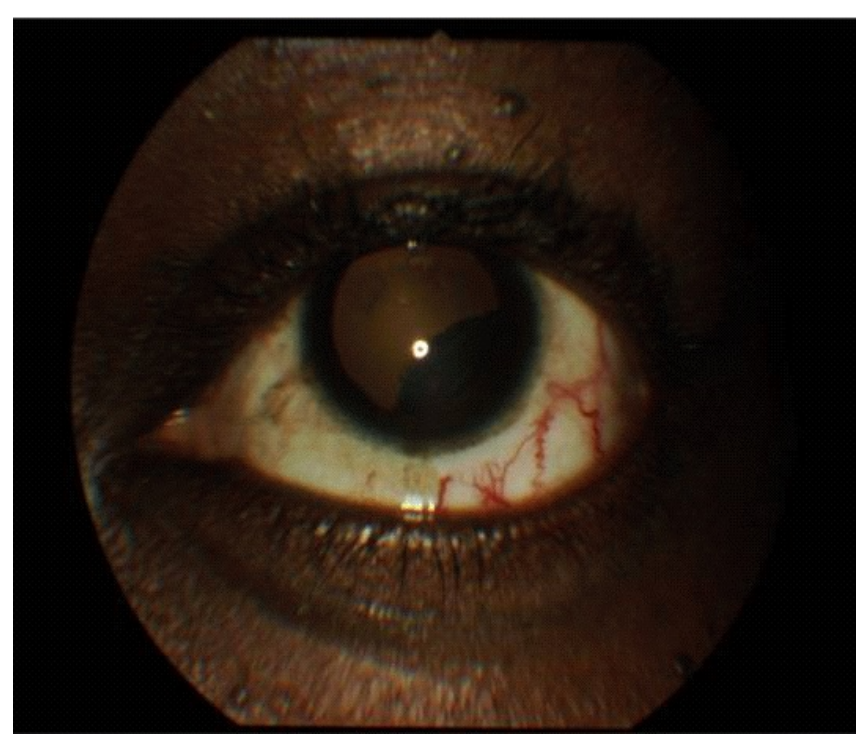

Figure 1 showing a widely dilated pupil of the left eye with a dark brownish pigmented mass lesion extending from the infero-temporal portion of the globe. Also note the dilated and tortuous episcleral (sentinel) vessels in the temporal portion of the globe harbouring the tumour and the absence of similar vessels in the nasal half of the globe. 


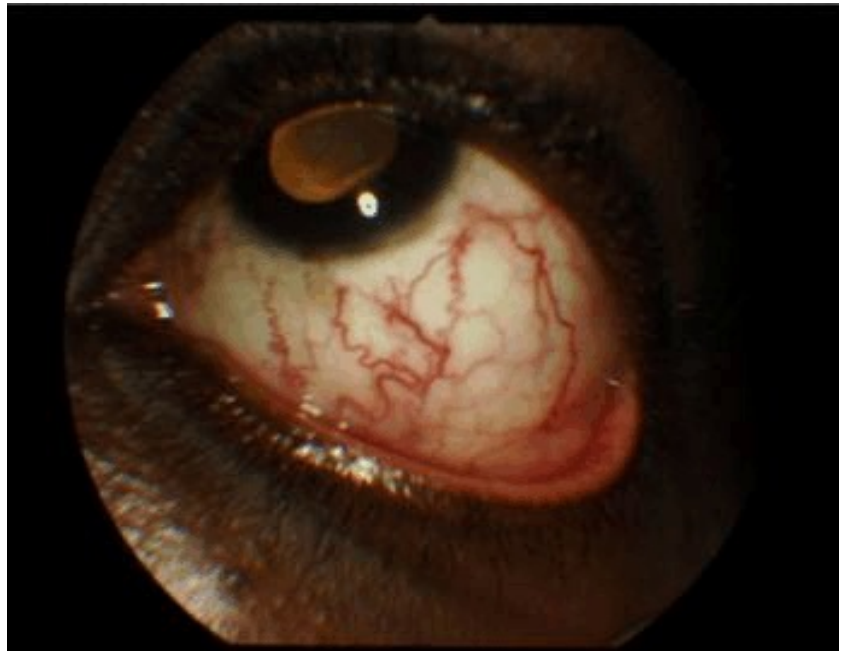

Figure 2. Temporal sentinel vessels exposed.

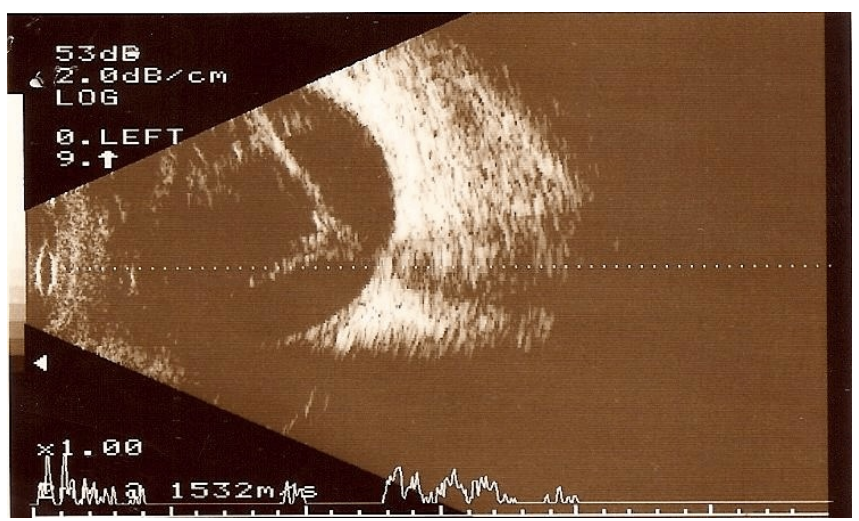

Figure 3. The left eye ultrasound B-scan showing a $V$ pattern closed funnel shaped retinal detachment.

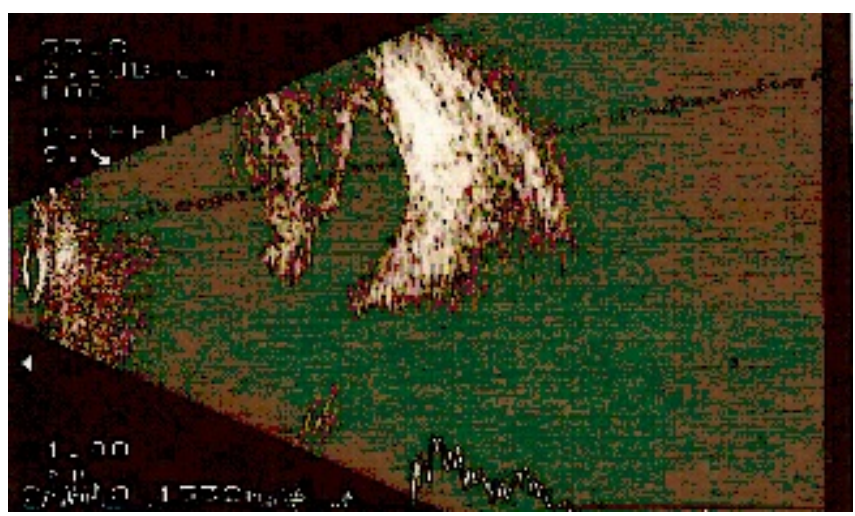

Figure 4. A horizontal scan of the anterior portion of the eye, showing an echogenic lesion anterior to the area of detached retina, in the region of the ciliary body and anterior vitreous.

She also presented with retinal detachment which is more commonly seen with a choroidal melanoma, but can also be seen in ciliary body melanoma. A retinal detachment is seen more often in situations involving a posterior extension of the ciliary body mass. Associated retinal detachment in a case of uveal mass further suggests a malignant potential. Uveal melanomas are associated with exudative retinal detachment. The possibility of an exudative retinal detachment with a uveal tumour was not considered at the time of initial presentation and examination because of the rarity of the condition. The fact that an obvious retinal break is not detected in a case of retinal detachment should always raise a suspicion of a non rhegmatogenous retinal detachment.

It is noteworthy that upon presentation, intraocular pressure in this patient was right eye $18 \mathrm{mmHg}$ and left eye $16 \mathrm{mmHg}$. This finding is unusual in a case of a primary rhegmatogenous retinal detachment in which the eye with the retinal detachment should have a hypotony, or have a much lower intraocular pressure than the other eye. The seemingly normal left eye intraocular pressure may have been as a result of the intraocular tumour. Gonioscopic evaluation of the left eye at presentation to rule out tumour invasion of the anterior chamber angle would have been essential.

It is important to consider other differential diagnoses of a ciliary body mass. These include a ciliary body cyst, melanocytic naevus, melanocytoma, metastatic tumours, medulloepithelioma (diktyoma), adenoma, adenocarcinoma and haemangioma. ${ }^{2}$

To make a definitive diagnosis in this case, a histological evaluation after enucleation can confirm the diagnosis and evaluate the prognosis. The patient declined to have an enucleation even though the visual acuity in the eye was no light perception.

An anterior chamber paracentesis for the aspiration of aqueous and cytological studies could increase the risk of extraocular spread, assuming the tumour is malignant. This technique was considered potentially harmful. After due consultation with an experienced ocular oncologist, it was agreed that enucleation of the globe would be the best line of management. The patient, however, declined surgery. This illustrates the challenge the clinician is bound to face convincing patients on the necessity to enucleate the globe in an eye harboring a mass lesion.

\section{CONCLUSION}

A 33-year-old lady presented with a left eye ciliary body mass having features suggestive of malignancy.

We are not aware of any other report of a ciliary body mass lesion with a likely diagnosis of melanoma in a Nigerian. This tumour is even rarer than its choroidal counterpart in published literature. The size and rate of growth of the tumour, the sentinel episcleral vessels, and the findings on B-scan ultrasonography, are all suggestive of 
malignancy. She presented with a total retinal detachment, suggesting that a non rhegmatogenous retinal detachment most likely secondary to the malignant melanoma can indeed present to our clinics. It is important to painstakingly search for the offending retina break in every case of a retinal detachment, and where no break is seen, a non rhegmatogenous retinal detachment and the possible differentials, including an intraocular tumour should be considered.

\section{REFERENCES}

1. Rennie I. Uveal melanoma. In: Taylor RH, Shah P, Murray PI, Burdon A, editors. Key Topics in Ophthalmology $2^{\text {nd }}$ edition. Oxford: BIOS Scientific Publishers 2001; 333-336.

2. Enrique GE, Mauricio EP. Melanoma, Ciliary Body; emedicine/WebMD Specialties; www.emedicine.com/ $\mathrm{OPH} /$ topic $404 \mathrm{htm}$

3. Nik NA, Glero WB, Zimmerman LE. Malignant melanoma of the choroid in the nevus of ota of a black patient. Arch Ophthalmol 1982; 100(10): 1641-3.

4. Margo CE, Mclean IW, Margo CA. Malignant melanoma of the choroid and ciliary body in black patients. Arch Ophthalmol 1984; 102 (1): 77-9.
5. Kheterpal S, Shields JA, Shields CL, De Potter P, Ehya H, Eng KY. Choroidal melanoma in an African-American albino. Am J Ophthalmol 1996; 122(6): 901-3.

6. Bordon AF, Wray ML, Belfort R, McLean IW, Burnier M. Choroidal malignant melanoma in association with oculodermal melanocytosis in a black patient. $\mathrm{Br} J$ Ophthalmol 1995; 79(2): 191-2.

7. Shields JA, Shields CL, Naseripor M, Eagle RC, Miller J. Choroidal melanoma in a black patient with oculodermal melanocytosis. Retina 2002; 22(1): 126-8.

8. Al-Sadhan $Y$, Shawaf $S$, Tabbara K. Oculodermal melanosis with choroidal melanoma in a black patient: a case report. Eye 2006; 20(12): 1437-8.

9. Nzegwu MA, Waziri-Erameh MJ, Ukponmwan CU, Igbe AP. Ocular melanoma presenting as a case of panophthalmitis in a 78-year old Nigerian woman: an uncommon finding in Benin City. Nigerian Eur J Cancer Care (Engl) 2007; 16(5): 451-2.

10. Anonubi CC, Akinsola FB, Abdulkareem FB, Aribaba OT, Anyiam DC, Daramola AO, et al. Conjunctival melanoma in a 55-year old male Nigerian: a case report. Niger Postgrad Med J 2006; 13(4): 366-9.

11. Basic and Clinical Science Course; Ophthalmic Pathology and Intraocular Tumors, Section 4; San Francisco, the Foundation of American Academy of Ophthalmology, p. 164. 